\title{
Characterization of Apolipoprotein A-I- and A-II-containing Lipoproteins in a New Case of High Density Lipoprotein Deficiency Resembling Tangier Disease and Their Effects on Intracellular Cholesterol Efflux
}

\author{
Marian C. Cheung, Armando J. Mendez, Anitra C. Wolf, and Robert H. Knopp \\ Department of Medicine, School of Medicine, University of Washington, Seattle, Washington
}

\begin{abstract}
A 48-yr-old Caucasian female of central European origin (subject IM) with low plasma cholesterol and normal plasma triglyceride (TG) had extremely low apo A-I (6 mg/dl), A-II (5 $\mathrm{mg} / \mathrm{dl}$ ), and HDL cholesterol ( $2 \mathrm{mg} / \mathrm{dl}$ ) levels. She had most of the clinical symptoms typically associated with Tangier disease, including early corneal opacities, yellow-streaked tonsils, hepatomegaly, and variable degrees of peripheral neuropathy, but had no splenomegaly. She had a myocardial infarction at age 46. Since HDL are postulated to be involved in the transport of excess cholesterol from peripheral tissues to the liver for degradation, and the ability of an HDL particle to promote cellular cholesterol efflux appears to be related to its density, size, and apo A-I and A-II contents, we isolated and characterized the HDL particles of this patient and all her first degree relatives (mother, a brother, and two children). The plasma A-I, A-II, and HDL cholesterol levels of all five relatives were either normal or high. Using anti-A-I and anti-A-II immunosorbents, we found three populations of particles in IM: one contained both apo A-I and A-II, Lp(AI w AII); one contained apo A-I but no A-II, Lp(AI w/o AII); and the third (an unusual one) contained apo A-II but no A-I, Lp(AII). Two-thirds of her plasma A-I and A-II existed in separate HDL particles, i.e., in $\operatorname{Lp}(\mathrm{AI} w / 0 \mathrm{AII})$ and $\mathrm{Lp}$ (AII), respectively. Only Lp (AI w AII) and Lp(AI w/o AII) were present in the plasma of the relatives. All three populations of the patient's HDL particles had a normal core/surface lipid ratio, but the cores were enriched with TG. The apo A-I-containing particles, however, were considerably smaller and contained much less lipid than Lp(AII). Despite these unusual physicochemical characteristics, the apo A-I-containing particles and Lp(AII) were effective suppressors of intracellular cholesterol esterification in cholesterol-loaded human skin fibroblast. The patient's plasma apo $D$ and lecithin cholesterol acyltransferase levels were reduced, with an increased proportion located in nonHDL plasma fractions. These findings are discussed in light of Tangier disease and other known HDL-deficiency cases, and
\end{abstract}

Address correspondence to Marian C. Cheung, Ph. D., Northwest Lipid Research Laboratories, University of Washington, 2121 N. 35th Street, Seattle, WA 98103. Dr. Mendez's current address is Cardiovascular Research Center, Massachusetts General Hospital, 149 13th Street, Charlestown, MA 02129.

Received for publication 16 May 1991 and in revised form $12 \mathrm{Au}$ gust 1992.

J. Clin. Invest.

(C) The American Society for Clinical Investigation, Inc.

$0021-9738 / 93 / 02 / 0522 / 08 \quad \$ 2.00$

Volume 91, February 1993, 522-529 the role of HDL in the maintenance of cell cholesterol homeostasis. (J. Clin. Invest. 1993.91:522-529.) Key words: cell cholesterol esterification $\bullet$ high density lipoprotein subpopulations - immunoaffinity chromatography • coronary artery disease • lecithin cholesterol acyltransferase

\section{Introduction}

Epidemiological studies have repeatedly demonstrated an inverse relationship between HDL and the risk of coronary artery diseases $(C A D)^{1}(1,2)$. The biochemical mechanism(s) behind this observed relationship is still unknown but is believed to be related to the role of HDL in promoting the transport of cholesterol from peripheral tissues to the liver for catabolism (reverse cholesterol transport) (3), thus preventing the accumulation of excess cellular cholesterol and the development of foam cells. Although this putative role of HDL in reverse cholesterol transport has not been proven conclusively, this concept is supported by many of the clinical features found in patients with HDL deficiency (4). Furthermore, in vitro studies have shown that HDL can promote cellular cholesterol efflux $(5,6)$.

Human HDL represents a population of particles that are heterogeneous in physical characteristics and in their lipid and protein composition. These particles are conventionally separated into two major density subfractions, $\mathrm{HDL}_{2}$ and $\mathrm{HDL}_{3}$, by ultracentrifugation. Using antibodies specific for apo A-I and A-II, HDL particles that differ in their apo A-I and A-II contents have been isolated $(7,8)$. In normal human plasma, HDL is comprised of two major populations of particles, both contain apo A-I but only one contains apo A-II: Lp(AI w AII) and $\mathrm{Lp}$ (AI w/o AII) (7-9). Recently, we identified a patient with HDL cholesterol and apo A-I levels below the fifth percentile of control population. Her plasma contained, besides $\mathrm{Lp}(\mathrm{AI} w \mathrm{AII})$ and $\mathrm{Lp}$ (AI w/o AII ), significant amounts of an unusual population of HDL particles with apo A-II as their major protein component: Lp(AII). Apo A-I was not detectable in these particles. This patient developed CAD at age 46. Since little is known regarding the characteristics of apo A-Iand A-II-containing lipoproteins in HDL deficiency and their ability to promote cellular cholesterol efflux, we isolated and characterized the three populations of HDL particles in this patient and studied their ability to promote intracellular cholesterol efflux. This report describes our findings and discusses them in relationship to Tangier disease and to our understand-

1. Abbreviations used in this paper: $\mathrm{CAD}$, coronary artery disease; $\mathrm{CE}$, cholesteryl ester; DS-Mg, dextran sulfate-magnesium; FC, free cholesterol; LCAT, lecithin cholesterol acyltransferase; LPL, lipoprotein lipase; PL, phospholipid; TG, triglyceride. 
ing of HDL subpopulations and their role in reverse cholesterol transport.

\section{Methods}

Subjects. Patient IM is a 48 -yr-old Caucasian female of central European origin. She is $5.0 \mathrm{ft}$ tall and weighs $93.5 \mathrm{lbs}$. She had a heart attack with no prior warning at age 46 , and coronary bypass surgery was performed shortly after the episode. She was referred to the Northwest Lipid Research Clinic (NWLRC) 2 yr after the bypass surgery because of her extremely low (2-3 mg/dl) HDL cholesterol level. On physical examination, she displayed an erythematous, scaling dermatitis in the seborrheic area of the face. Her eyes showed fine corneal opacities on slit lamp examination characteristic of Tangier disease. The thyroid was normal but whitish-yellowish streaking was seen in both tonsillar fossae, right greater than left. The chest was clear, and the heart showed no cardiomegaly or point of maximum impulse. A grade 2 of 6 systolic ejection murmur in the aortic area was noted. The $\mathbf{2} 2$ was widely split with inspiration and there was a fourth heart sound gallop. She reported having chest tightness with exercise consistent with angina pectoris and shortness of breath on rapid walking consistent with congestive failure. The abdomen showed an $11-\mathrm{cm}$ liver that had a $1-\mathrm{cm}$ palpable edge. It was soft and nontender. No spleen was palpable. Abdominal ultrasound confirmed the hepatomegaly and normal spleen size. The extremities showed puffy nonpitting edema to the knees. The right dorsalis pedis pulse was palpable, the posterior tibial pulses were absent bilaterally. The popliteal pulse was present on the right and indeterminate on the left. Acrocyanosis of the toes was noted. The ankle jerks were absent and there was decreased pin and vibration sense in the great toes. At a repeat examination 2 yr later, the pin-prick awareness in the toes was improved but the ankle jerks were still absent.

The subject's family history of heart disease is difficult to assess because grandparents, aunts, and uncles died in Europe during World War II. However, her mother (MK, age 75) is alive and well with a normal total and HDL cholesterol, and her father died at age 76 of adult-onset diabetes. The 56-yr-old brother (PK) also has normal plasma and HDL cholesterol with ulcer disease treated with diet, but is asymptomatic for CAD. The patient has a 17-yr-old son (MM) and a 14-yr-old daughter (HM) with no known clinical disease. Her husband died of cancer at age 51 . The subject and her children habitually eat a low fat diet. There was no known consanguinity.

Plasma samples. Blood samples from the patient and her two children were obtained at the NWLRC. Venous blood was drawn from the antecubital vein into EDTA-containing Vacutainer tubes (BectonDickinson \& Co., Rutherford, NJ) after a 12-14-h overnight fast. Plasma was separated by low speed centrifugation at $4^{\circ} \mathrm{C}$, and sodium azide, gentamycin, and chloramphenicol were promptly added to final concentrations of $0.5,0.005$, and $0.01 \mathrm{~g} /$ liter, respectively. Fasting plasma samples from her mother and brother were air shipped in ice to Seattle. All HDL isolations commenced within $48 \mathrm{~h}$ after the blood was drawn.

Lipoprotein fractionation. Fractionation of the patient's plasma into VLDL, LDL, and HDL was performed as described (10). Briefly, plasma was subjected to ultracentrifugation at $105,000 \mathrm{~g}$ for $18 \mathrm{~h}$ without prior density adjustment. VLDL was recovered in the top fraction by the tube-slicing technique. LDL and HDL were recovered in the bottom fraction. HDL was separated from plasma by precipitation with dextran sulfate and magnesium (11). Lipid in each fraction was quantitated. LDL lipid was calculated as the difference between the $d$ 1.006 bottom fraction and the dextran sulfate-magnesium (DS-Mg) supernatant fraction. VLDL lipid was calculated as the difference between plasma lipid and the $d 1.006$ bottom fraction. In the relatives, HDL was separated from VLDL and LDL by DS-Mg. Total plasma cholesterol, triglyceride (TG), and cholesterol in the DS-Mg supernatant fraction were quantitated. The cholesterol content of VLDL and LDL were calculated as described by Friedewald et al. (12).

Isolation of $H D L$ particles. HDL particles differing in their apo A-I and A-II contents were isolated from the patient's plasma obtained under two occasions $2 \mathrm{yr}$ apart. Particles from the first isolation were used for detailed physicochemical characterization. Those from the second isolation were used for intracellular cholesterol efflux studies.

The first isolation, and isolations from plasma samples of the relatives, followed an established two-step immunoaffinity chromatography procedure $(7,8)$ with the following modifications. Plasma aliquots ( $20 \mathrm{ml}$ from the patient, and $2.5-5 \mathrm{ml}$ from her relatives) were sequentially adsorbed with DS cellulose, anti-A-II-Sepharose CL 4B, and anti-A-I-Sepharose CL $4 B$ to respectively remove all apo B-containing lipoproteins (13), apo A-II-containing lipoproteins, and apo A-I-containing lipoproteins that do not have any apo A-II. Nonadsorbed proteins were washed with $0.01 \mathrm{M}$ Tris- $\mathrm{HCl}$ buffer, $\mathrm{pH} 7.4$, containing $0.15 \mathrm{M} \mathrm{NaCl}, 1 \mathrm{mM}$ EDTA, and $0.05 \%$ sodium azide. Lipoproteins bound to DS cellulose and the immunosorbents were eluted with $3 \mathrm{M}$

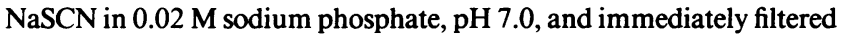
through a column packed with Sephadex G-25 (Pharmacia LKB Biotechnology Inc., Piscataway, NJ) to remove the thiocyanate. In the patient, but not her relatives, the lipoproteins eluted from the anti-A-II immunosorbent contained three times more apo A-II than A-I. These lipoproteins were further adsorbed with the anti-A-I immunosorbent to separate particles, which contained both apo A-II and A-I from those unusual ones that contained apo A-II but no A-I. Nonadsorbed proteins and adsorbed lipoproteins were concentrated by concentrator (MicroConfilt; Bio-Molecular Dynamics, Beaverton, OR) for further studies. All isolation and concentration processes were performed at $4^{\circ} \mathrm{C}$.

In the second isolation, $12 \mathrm{ml}$ of the subject's plasma was sequentially adsorbed with DS cellulose, anti-A-I-Sepharose and anti-A-IISepharose. By reversing the sequence of the immunosorbents, all apo A-I-containing lipoproteins, i.e., $\mathrm{Lp}(\mathrm{AI} w \mathrm{AII})$ and $\mathrm{Lp}$ (AI w/o AII) bound to the anti-A-I immunosorbent whereas only $\mathrm{Lp}$ (AII) bound to the anti-A-II column. These lipoproteins were desorbed from the immunosorbent as described above and were further subjected to heparin Sepharose chromatography to remove apo E-containing lipoproteins (14) before cell cholesterol esterification studies.

Cell cholesterol esterification studies. Normal skin fibroblasts were cultured from explants of punch biopsies of skin from the inner thigh of normal volunteers as previously described (15). Fibroblasts between passage 5 and 15 were grown to confluence (7-10 d) before use. Confluent cells were rinsed twice with PBS containing $1 \mathrm{mg} / \mathrm{ml} \mathrm{BSA}$, and cholesterol-loaded by incubation for $48 \mathrm{~h}$ with serum-free DME containing $2 \mathrm{mg} / \mathrm{ml} \mathrm{BSA}$ and $50 \mu \mathrm{g} / \mathrm{ml}$ (nonlipoprotein) cholesterol. After loading with cholesterol, cells were rinsed three times with PBS containing $2 \mathrm{mg} / \mathrm{ml} \mathrm{BSA}$, and fresh DME containing $1 \mathrm{mg} / \mathrm{ml} \mathrm{BSA}$ was added for $16 \mathrm{~h}$ to allow equilibration of cholesterol pools. Subsequently, cells were rinsed and incubated with DME containing $1 \mathrm{mg} /$ $\mathrm{ml} \mathrm{BSA}$ and various amounts of HDL particles for $16 \mathrm{~h}$. The medium was then removed and the cells were rinsed once with PBS to remove the added HDL. Intracellular pool of cholesterol available for esterification by acyl-CoA: cholesterol acyltransferase were estimated by incubating cells with DME containing $9 \mu \mathrm{M}{ }^{14} \mathrm{C}$-oleate complexed with albumin for $1 \mathrm{~h}$. After incubation, the cells were chilled on ice, washed two times with ice-cold PBS/BSA then twice with ice-cold PBS. The cells were extracted immediately and free and esterified cholesterol were separated by thin layer chromatography and determined by scintillation counting as previously described (16).

Analytical procedures. Total and unesterified cholesterol (C, FC), phospholipid (PL), and TG in the plasma and lipoprotein fractions were measured by enzymatic methods (17). Apo A-I, A-II, B, D, and lecithin cholesterol acyltransferase (LCAT) mass were quantitated by specific immunoassays (18-22). Plasma LCAT activity was measured using the apo A-I proteoliposome common substrate method (23). The protein composition of the isolated HDL particles was examined by nonreducing SDS 7-20\% gradient PAGE according to the method of Laemmli (24). HDL particle sizes were determined by nondenaturing gradient PAGE using precast 4-30\% gels (Pharmacia LKB Biotechnology Inc.) (25) with electrophoresis carried out to 1,500 and to 3,000 $\mathrm{V} h$. The former prevented the loss of particles $<7.0 \mathrm{~nm}$ in Stokes 
diameter. The latter allowed the larger particles and the calibration proteins to migrate to equilibrium. Proteins in the SDS and nondenaturing gels were visualized with $0.1 \%$ Coomassie blue R-250 and G-250, respectively. Gels were scanned and integrated with the Gelscan XL software (2400; Pharmacia LKB Biotechnology Inc.). Chemical cross-linking studies were performed with dimethylsuberimidate according to the method of Swaney and O'Brien (26). The crosslinked products were delipidated and analyzed with SDS-PAGE as described (27).

\section{Results}

Lipid and apo profile. The patient's lipid profile determined under two different occasions 2 yr apart had an unusually low total plasma cholesterol attributable to her extremely low HDL cholesterol (Table I). Although her plasma TG was normal, most of it $(60 \%)$ was located in LDL rather than VLDL. The lipid composition of the DS-Mg supernatant fraction, usually considered representative of HDL, was quite different from that of normal. Specifically, there was as much TG as cholesterol, and the PL content was nine times more than the cholesterol. The HDL cholesterol levels in the patient's relatives were between the 10th and 50th percentiles for age- and sexmatched controls (28). Their overall lipid profiles and the lipid composition of the DS-Mg supernatant (HDL) fraction were normal although the HDL fraction of PK, who had elevated plasma TG, was TG enriched.

Consistent with her unusually low HDL cholesterol level, the plasma apo A-I and A-II levels of the patient were $\sim 4$ and $14 \%$ of controls, respectively (Table II). Thus her plasma A-I/ A-II ratio was considerably lower than that in controls. Apo D and LCAT, two other proteins normally found in HDL, were also reduced ( Table II), but to a lesser extent (30-40\% of controls). Plasma LCAT activity $\left(20.4 \mathrm{nmol} \cdot \mathrm{h}^{-1} \cdot \mu \mathrm{g} \mathrm{LCAT}{ }^{-1}\right)$ measured with the exogenous A-I proteoliposome substrate was comparable to $\mathrm{MK}\left(20.7 \mathrm{nmol} \cdot \mathrm{h}^{-1} \cdot \mu \mathrm{g} \mathrm{LCAT}^{-1}\right)$ and PK $\left(22.0 \mathrm{nmol} \cdot \mathrm{h}^{-1} \cdot \mu \mathrm{g} \mathrm{LCAT}^{-1}\right)$. With the exception of an unusually high apo A-II concentration in PK, the plasma levels of these proteins in the patient's relatives were within the normal range for an adult population. Plasma apo B levels were normal in all subjects.

Characteristics of HDL particles. With sequential immunoaffinity chromatography, three populations of HDL particles were isolated from the plasma of the patient: one contained both apo A-I and A-II, Lp(AI w AII); one contained apo A-I but not A-II, Lp(AI w/o AII); and the third (an unusual one), contained apo A-II but not A-I, Lp(AII). Two thirds of the plasma apo A-I and A-II were found in $\operatorname{Lp}(\mathrm{AI}$ w/o AII $)$ and $\mathrm{Lp}$ (AII), respectively. Thus, the majority of the apo A-I and A-II in the patient existed in separate HDL particles, and only $25 \%$ of plasma A-I and $20 \%$ of plasma A-II were located in $\mathrm{Lp}$ (AI w AII). In the patient's relatives, both $\mathrm{Lp}$ (AI w AII) and $\mathrm{Lp}$ (AI w/o AII) were present, with the majority of apo A-I (60-87\%) located in particles with apo A-II. There was no evidence of any significant amount of $\mathrm{Lp}$ (AII), and $\sim 1 \%$ of apo A-I and A-II were detected in the materials bound to DS cellulose (the DS fraction). This DS fraction contained essentially all the apo B-containing lipoproteins. However, in the patient, an abnormally high $7 \%$ of plasma A-I and $12 \%$ of plasma A-II were recovered in this fraction.

The distribution of apo D and LCAT among the various HDL particles was studied. In all five subjects, relatively more apo D was associated with apo A-II-containing particles whereas LCAT was found mostly in particles without apo A-II (Table III). In the patient's relatives, the HDL-associated apo D and LCAT represented 84 and $93 \%$ of total apo D and LCAT in plasma, respectively. In contrast, over $40 \%$ of the patient's apo D, and $30 \%$ of LCAT were in the non-HDL plasma fractions. In the patient and in MK and PK, non-HDL apo D was located in the DS fraction as well as the apo A-I-, A-II-, and B-free plasma, i.e., lipoprotein-deficient plasma. In MM and HM, non-HDL apo D was detected only in the DS fraction. In all subjects, nearly $90 \%$ of the non-HDL LCAT was recovered in the lipoprotein-deficient plasma.

The protein composition of the HDL particles was also examined by SDS-PAGE. As shown in Fig. 1, no protein band corresponding to apo A-II was detected in $\mathrm{Lp}$ (AI w/o AII), and the patient's $L p(A I I)$ did not contain any protein with a molecular weight comparable to normal A-I. Although, a distinct protein that migrated slightly ahead of normal apo A-I was observed, when the SDS gel was electrophoretically transferred to a nitrocellulose membrane according to the method of Towbin et al. (29), this protein band did not react with polyclonal antibodies to apo A-I, A-II, or D (data not shown). Furthermore, a comparable protein band was not detected in the Lp(AII) isolated from the second plasma sample (data not shown). Proteins at molecular weight positions comparable to normal apo A-I and A-II were detected in the Lp(AI w A-II) of the patient and her relatives. Examples are shown in Fig. 1. As we and others have observed, proteins larger than apo A-I and smaller than apo A-II were seen in all HDL particles $(30,31)$.

Table I. Lipid Profile of Patient and Family

\begin{tabular}{|c|c|c|c|c|c|c|c|c|c|c|c|c|c|c|c|c|c|}
\hline \multirow[b]{2}{*}{ Subject } & \multirow[b]{2}{*}{ Age/sex } & \multicolumn{4}{|c|}{ Plasma } & \multicolumn{4}{|c|}{ VLDL } & \multicolumn{4}{|c|}{ LDL } & \multicolumn{4}{|c|}{ HDL } \\
\hline & & TC & FC & TG & PL & TC & FC & TG & PL & TC & FC & TG & PL & TC & FC & TG & PL \\
\hline & & \multicolumn{16}{|c|}{$m g / d l$} \\
\hline IM (1st visit) & $49 / F$ & 102 & 28 & 120 & 115 & 14 & 5 & 42 & 21 & 86 & 22 & 75 & 76 & 2 & $<1$ & 3 & 18 \\
\hline IM (2nd visit) & $51 / \mathrm{F}$ & 89 & 24 & 153 & 123 & 13 & 4 & 61 & 8 & 73 & 18 & 89 & 98 & 2 & 1 & 2 & 17 \\
\hline MM & $17 / \mathrm{M}$ & 126 & 29 & 59 & 150 & 12 & NA & NA & NA & 75 & NA & NA & NA & 39 & 7 & 8 & 91 \\
\hline HM & $14 / F$ & 141 & 33 & 62 & 170 & 12 & NA & NA & NA & 85 & NA & NA & NA & 43 & 7 & 10 & 101 \\
\hline MK & $75 / \mathrm{F}$ & 192 & 41 & 81 & 205 & 16 & NA & NA & NA & 135 & NA & NA & NA & 41 & 6 & 2 & 91 \\
\hline PK & $56 / \mathrm{M}$ & 178 & 42 & 249 & 292 & 50 & NA & NA & NA & 82 & NA & NA & NA & 46 & 7 & 27 & 172 \\
\hline
\end{tabular}

NA, not analyzed. F, female; M, male. FC, free cholesterol. 
Table II. Plasma Apolipoprotein and LCAT Levels of Patient and Family

\begin{tabular}{lrrrrc}
\hline \multicolumn{1}{c}{ Subject } & AI & AII & B & D & LCAT \\
\hline & \multicolumn{5}{c}{$m g / d l$} \\
IM (1st visit) & 6 & 5 & 94 & 2.2 & \multicolumn{1}{c}{1.8} \\
IM (2nd visit) & 6 & 4 & 94 & 2.0 & 2.4 \\
MM & 104 & 26 & 94 & 4.5 & 6.1 \\
HM & 122 & 34 & 108 & 4.4 & 3.3 \\
MK & 125 & 31 & 118 & 4.4 & 8.0 \\
PK & 147 & 54 & 108 & 7.9 & 7.4 \\
Control & & & & & \\
$\quad$ Male & $120 \pm 20$ & $33 \pm 5$ & $102 \pm 24$ & $6.2 \pm 1.1$ & $5.6 \pm 0.9$ \\
Female & $135 \pm 26$ & $36 \pm 6$ & $98 \pm 23$ & $5.9 \pm 1.3$ & $5.9 \pm 1.1$ \\
& & & & & \\
\hline
\end{tabular}

Control values obtained with the same immunoassays for adult populations (see references 18-22).

The larger ones were predominantly located in particles without apo A-II whereas those with molecular weights similar to the apo Cs were mostly found in Lp(AI w AII).

The lipid composition of the various HDL particles are shown in Table IV. In the relatives the composition was similar to that reported for other subjects of comparable plasma lipid levels $(7,30,32)$. Although IM had a normal plasma TG level, her HDL were enriched in TG and depleted of cholesteryl ester (CE). Furthermore, her two apo A-I-containing lipoprotein particles had a much lower lipid/protein ratio than that of her relatives, suggesting that they were denser HDL particles. In contrast, her Lp(AII) contained nearly equal amounts of lipid and protein, a ratio approaching the two populations of A-Icontaining particles of her relatives.

Agarose gel electrophoresis was performed on the patient's HDL particles. Due to the small amount of materials available, and probably to the low lipid content of some of the particles, we were only able to see, with certainty, alpha migrating Lp(AII) when the gel was stained for lipid with Sudan black. Staining the gel for protein with Coomassie G-250 revealed alpha and prebeta materials in both $\mathrm{Lp}(\mathrm{AI} \mathrm{w} / \mathrm{o} \mathrm{AII})$ and Lp(AI w AII) (data not shown).

The particle sizes of the three populations of HDL were studied by nondenaturing gPAGE. Each population of HDL

Table III. Distribution of Apo D and LCAT in Plasma

A-I-, A-II-,

Subject $\operatorname{Lp}(A I$ w AII) $\quad$ Lp(AI w/o AII) Lp(AII) Ds fraction B-free plasma

\begin{tabular}{rlrrrrr}
\hline ApoD & IM & 26 & 10 & 23 & 18 & 23 \\
& MM & 76 & 17 & - & 7 & ND \\
& HM & 71 & 21 & - & 8 & ND \\
& MK & 72 & 14 & - & 8 & 6 \\
& PK & 85 & 6 & - & 5 & 4 \\
LCAT & IM & 1 & 66 & 2 & 4 & 27 \\
& MM & 11 & 73 & - & 2 & 14 \\
& HM & 12 & 72 & - & $<1$ & 16 \\
& MK & 23 & 63 & - & 2 & 12 \\
& PK & 22 & 62 & - & $<1$ & 16 \\
\end{tabular}

All numbers represent percent of total D or LCAT mass in the plasma of each subject. The Ds fraction contains all apo B-containing lipoproteins. ND, not detectable.

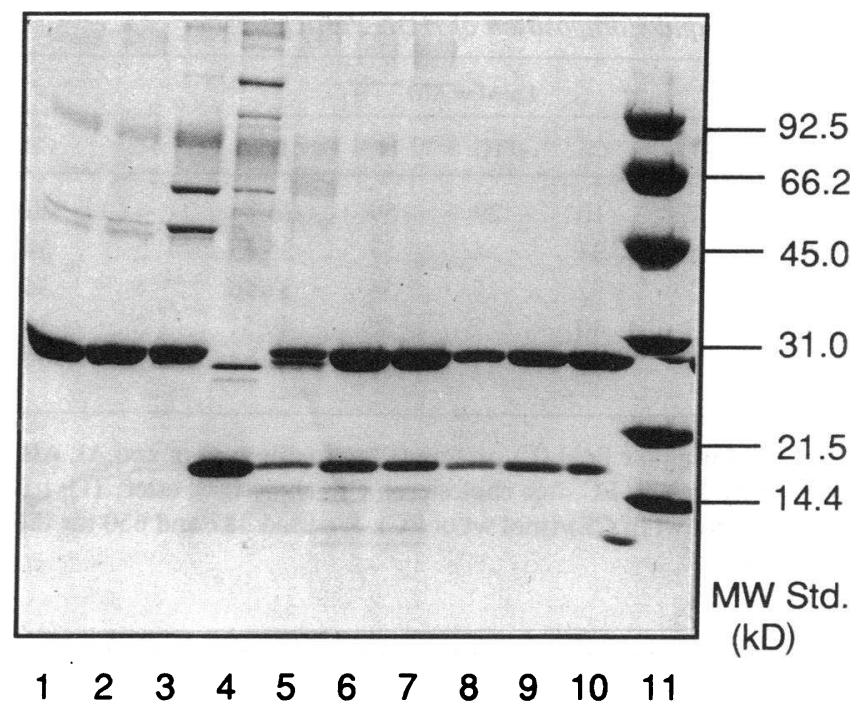

Figure 1. Nonreducing SDS (7-20\%) PAGE of HDL particles. Lanes 3 , 4, and 5 are the $\mathrm{Lp}$ (AI w/o AII), Lp(AII), and Lp(AI w AII) of patient IM, respectively. Also shown for comparison are the $\mathrm{Lp}(\mathrm{AI}$ w/o AII) (lanes 1 and 2) and the Lp(AI w AII) (lanes 6 and 7) of $\mathrm{HM}$ and MM. Lanes $8-10$ are a mixture of purified A-I $(2,4$, and 6 $\mu \mathrm{g}$, respectively) and A-II $(0.8,1.6$, and $2.4 \mu \mathrm{g}$, respectively); lane 11 shows low molecular weight standards from Bio-Rad Laboratories (Richmond, CA).

contained particles of mixed sizes (Figs. 2 and 3). However, the overall size profiles of the patients' $L p$ (AI w AII) and $\mathrm{Lp}(\mathrm{AI} w / \mathrm{o}$ AII $)$ were much smaller than those of her relatives. On the basis of densitometry, the majority (83-87\%) of the relatives' $L p(A I$ w AII) and $L p(A I$ w/o AII) were located within the 8.2-17.0-nm Stokes diameter interval. Examples are shown in Fig. 2. Conversely, three quarters of these particles in patient IM were $<8.2 \mathrm{~nm}$ with substantial amounts $(47-52 \%)$ $<7.0 \mathrm{~nm}$. Although $42 \%$ of the patient's Lp(AII) particles were located in the size region $<8.2 \mathrm{~nm}$, only $4 \%$ of those particles were $<7.0 \mathrm{~nm}$, and a distinct size species with a peak Stokes diameter of $8.0 \mathrm{~nm}$ was observed (Fig. $3 \mathrm{~A}$ ). Cross-linking of $\mathrm{Lp}$ (AII) with dimethylsuberimidate followed by delipidation and SDS-PAGE showed that the majority of Lp(AII) particles likely contained proteins with a total molecular weight equivalent to four molecules of apo A-II (Fig. $3 \mathrm{~B}$ ). (One molecule of A-II is defined as two identical peptides of 77 amino acids.)

Effect of HDL intracellular cholesterol efflux. The ability of the patient's $L p$ (AII) and total apo A-I-containing lipoproteins, $\mathrm{Lp}(\mathrm{AI})$, i.e., combination of $\mathrm{Lp}(\mathrm{AI} \mathrm{w}$ AII ) and $\mathrm{Lp}(\mathrm{AI}$ w/o AII), to promote intracellular cholesterol efflux was measured by the ability of these lipoproteins to decrease esterification of cell cholesterol in cholesterol-loaded cultured human fibroblasts. Both $L p(A I)$ and $L p($ AII ) reduced cell cholesterol esterification in a dose-dependent manner (Fig. 4). Furthermore, the effectiveness of these particles were comparable to the $\mathrm{Lp}(\mathrm{AI})$ from a normolipidemic subject at similar total protein concentration and apparently more effective when normalized for A-II concentration.

\section{Discussion}

This report describes the characteristics of the HDL particles in a new case of apo A-I deficiency. The patient's overall plasma 


\begin{tabular}{|c|c|c|c|c|c|c|c|c|c|c|c|c|c|c|c|}
\hline \multirow[b]{2}{*}{ Subject } & \multicolumn{5}{|c|}{ Lp(AI w AII) } & \multicolumn{5}{|c|}{ Lp(AI w/o AII) } & \multicolumn{5}{|c|}{ Lp(AII) } \\
\hline & FC & CE & TG & PL & $\mathrm{L} / \mathrm{P}^{*}$ & FC & $\mathrm{CE}$ & TG & PL & $\mathbf{L} / \mathbf{P}$ & $\mathrm{FC}$ & CE & TG & PL & $\mathbf{L} / \mathbf{P}$ \\
\hline IM & 2 & 10 & 29 & 59 & $26: 74$ & 4 & 20 & 20 & 56 & $25: 75$ & 4 & 18 & 17 & 61 & $46: 54$ \\
\hline $\mathbf{M M}$ & 5 & 34 & 4 & 57 & $55: 45$ & 5 & 34 & 5 & 56 & $54: 46$ & & & & & \\
\hline $\mathrm{HM}$ & 5 & 32 & 5 & 58 & $54: 46$ & 4 & 34 & 6 & 56 & $56: 44$ & & & & & \\
\hline MK & 3 & 40 & 7 & 50 & $49: 51$ & 3 & 36 & 14 & 47 & $50: 50$ & & & & & \\
\hline PK & 3 & 31 & 11 & 55 & $54: 46$ & 2 & 29 & 17 & 52 & $48: 52$ & & & & & \\
\hline
\end{tabular}

* In calculating the lipid (L)/protein (P) ratio, the sum of apo AI, AII, D, and LCAT mass was used as the protein mass. All numbers represent percent by weight. FC, free cholesterol; CE, cholesteryl ester; TG, triglyceride; PL, phospholipid. CE mass was calculated as (Total CH - FC) mass $X$ (mol wt of CE)/(mol wt of FC). We used 387 and 650 for the molecular weight of FC and CE, respectively.

lipid and lipoprotein concentrations were comparable to those reported for individuals homozygous for Tangier disease and familial apo A-I and C-III deficiency, but her apo A-I and A-II levels resembled more those of Tangier homozygotes $(4,33)$. Her extremely low HDL cholesterol content likely accounted for her unusually low total plasma cholesterol level. Although her plasma TG level was normal, it was disproportionately located in the LDL fraction, resulting in TG-rich LDL. An abnormal distribution of plasma TG between VLDL and LDL has also been observed in Tangier homozygotes $(4,33)$ and in a subject homozygous for the apo A-I ( $\mathrm{Glu}_{136}$-Lys) variant (34) but was not found in familial apo A-I and C-III deficiency (4, 35,36 ). On clinical grounds, IM also had most of the features seen in Tangier disease, including yellow streaked tonsils, early corneal opacities, hepatomegaly, and variable degrees of peripheral neuropathy. However, she did not have the splenomegaly

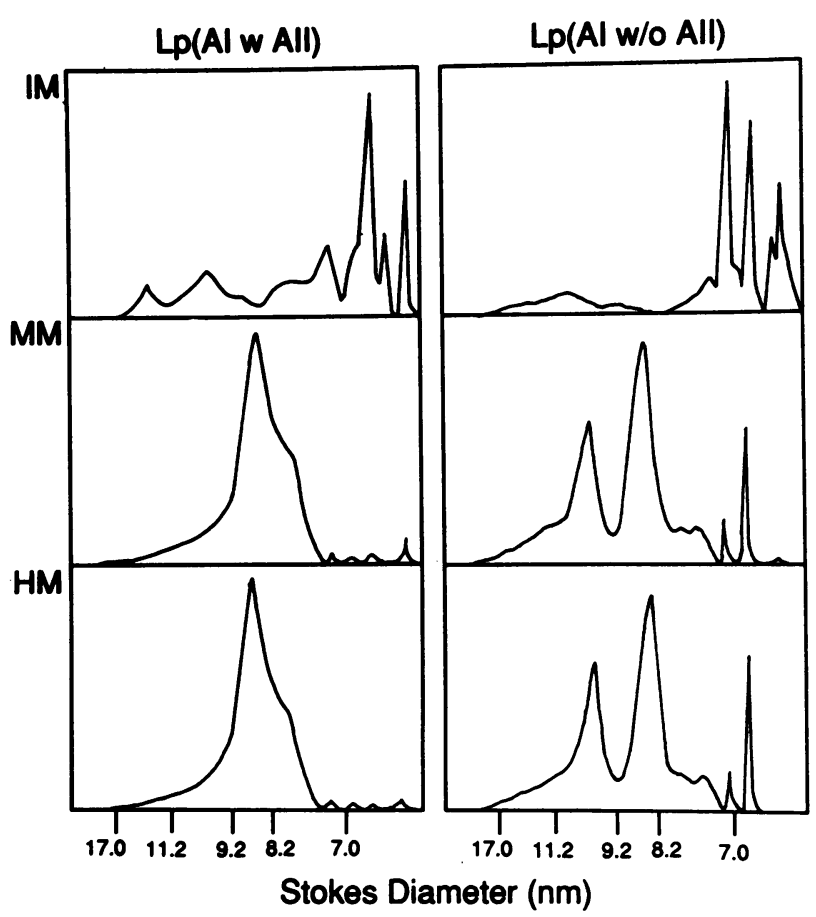

Figure 2. HDL particle size profiles. Densitometric scans after nondenaturing 4-30\% PAGE of patient IM's Lp(AI w AII) (left) and $\mathrm{Lp}(\mathrm{AI}$ w/o AII ) (right). Shown for comparison were the HDL particles of her children, MM and HM. reported in two thirds of Tangier cases (4). The gross lipid and apolipoprotein profiles of her four first-degree relatives appear normal, though many parameters of her two children were on the low side of normal range for an adult population. Whether this finding is related to the low fat diet of the family or that the children are heterozygous for the unknown maternal defect(s) that 'eads to HDL deficiency remains to be determined.

Since the molecular defects underlying the profound HDL deficiency in IM and in Tangier disease are not known, a definitive statement regarding whether IM has Tangier disease cannot be made. Nevertheless, the patient was found to have an increased ratio of pro to mature apo A-I and an increased amount of disialated apo E similar to that found in patients with Tangier disease (Rader, D., personal communication). Her apo A-I and A-II were indistinguishable from normal A-I and A-II in nonreduced SDS-PAGE. Thus, the primary defect in IM is different from that in two recently reported cases of apo A-I deficiency that involved a frameshift mutation (37) and a 45-bp deletion (38) in the apo A-I gene. In the patient with frameshift mutation, an A-I-A-II hetero dimer was detected by immunoblot of isoelectric focusing gel and mass spectrometry. In the patient with a 45-bp deletion, his apo A-I migrated slightly faster than normal A-I in SDS-PAGE. The normal free/esterified cholesterol in the patient's plasma showed that the cholesterol esterification function of LCAT was not significantly affected by the strikingly low level of A-I. Furthermore, the ability of her plasma to esterify exogenous cholesterol in proteoliposome containing apo A-I indicates that she does not have defects similar to Fish Eye disease (39). Posthe-
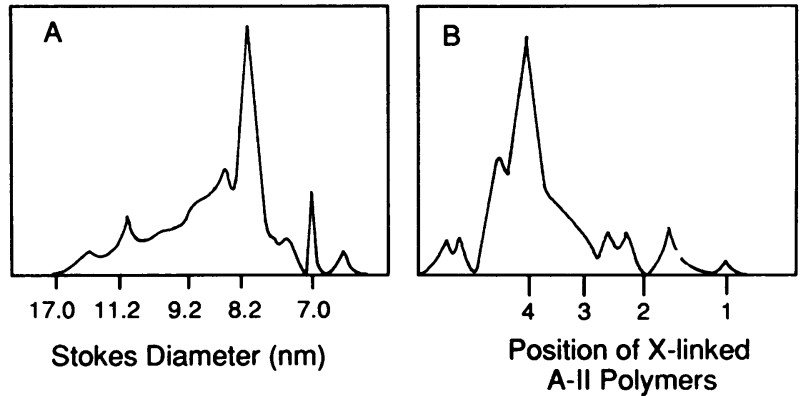

Figure 3. Particle size profile and protein molecular weight of the $\mathrm{Lp}$ (AII) of the patient. Densitometric scans of $(A) \mathrm{Lp}$ (AII) after nondenaturing 4-30\% PAGE and $(B)$ chemically cross-linked Lp(AII) upon SDS 4-30\% PAGE. 

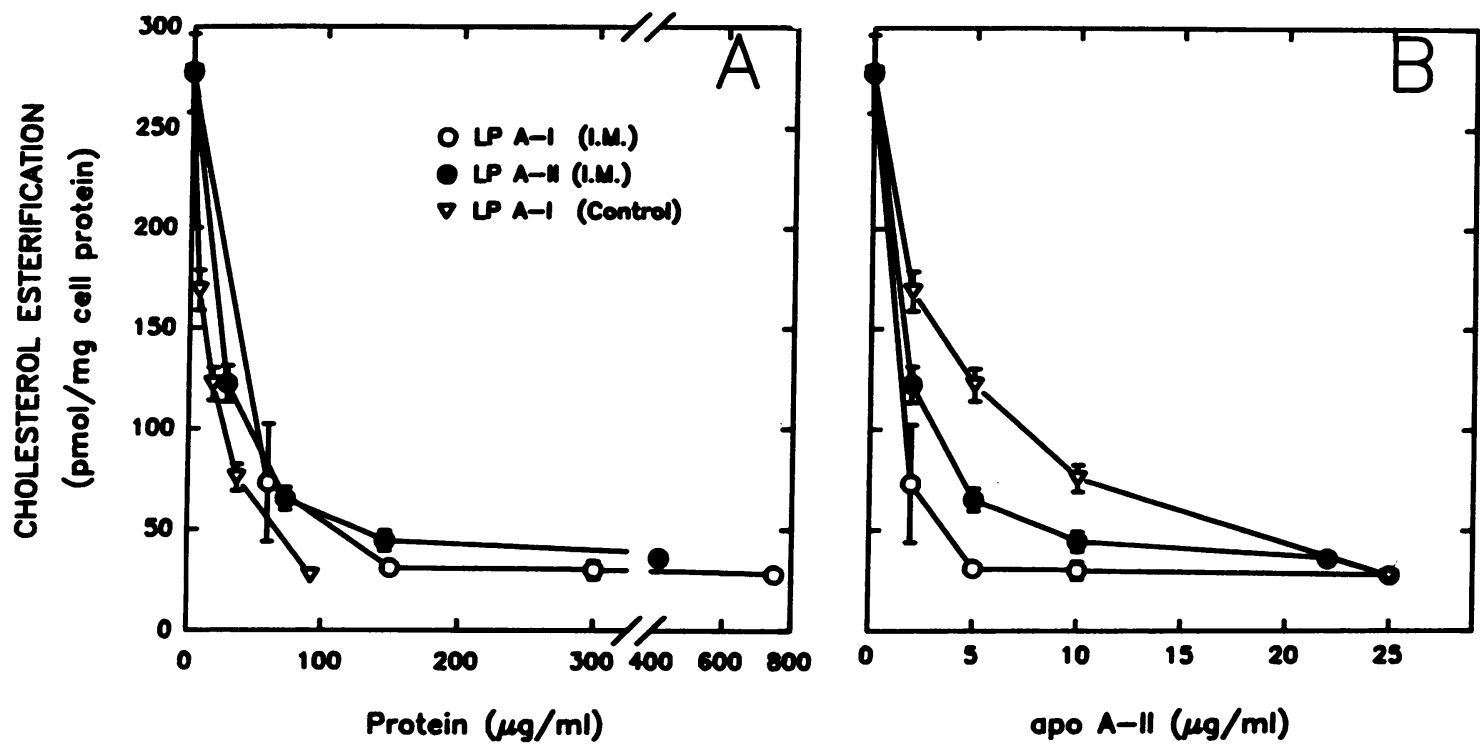

Figure 4. Effects of patient and control HDL fractions on cholesterol esterification in cholesterol-loaded fibroblasts. Cultured cells were incubated in medium containing $1 \mathrm{mg} / \mathrm{ml}$ BSA and the indicated concentrations of lipoproteins for $16 \mathrm{~h}$ followed by a 1 -h pulse incubation with medium containing $9 \mu \mathrm{M}{ }^{14} \mathrm{C}$-oleate. (The 16-h incubation allowed maximum depletion of acyl-CoA: cholesterol acyltransferase substrate pools). After incubation, cell lipids were extracted, separated by thin-layer chromatography, and radioactivity in CE was measured. Cholesterol esterification was quantitated as picomoles of ${ }^{14} \mathrm{C}$-oleate incorporated into $\mathrm{CE}$ per $\mathrm{mg}$ of cell protein. $(A)$ Values normalized for total protein content of lipoprotein fraction. $(B)$ Values normalized for apo A-II content of lipoprotein fraction.

parin plasma hepatic triglyceride lipase activity was also normal, 89 versus $90 \pm 40 \mathrm{nmol} / \mathrm{min}^{-1}$ per $\mathrm{ml}^{-1}$ for normal females. (hepatic triglyceride lipase activity was measured by Dr. J. D. Brunzell, University of Washington, Seattle, WA). Several apo A-I variants resulting from a single amino acid substitution or deletion have also been reported $(34,40-43)$. Their HDL cholesterol and apo A-I levels, however, ranged between subnormal to normal, and there was no evidence of $\mathrm{Lp}$ (A-II) in two A-I $\mathrm{I}_{\text {Milano }}$ carriers studied (27). Thus, it is unlikely that the molecular defect(s) in IM resembles any of these known cases of apo A-I variants. Taken together, the apo A-I deficiency in IM is likely due to rapid catabolism as in Tangier disease, and the possibility of a defect in the conversion of pro apo A-I to apo A-I cannot be ruled out.

Besides being deficient in quantity, the HDL present in the patient's plasma were abnormal in composition and size. In normal subjects and in the patients' relatives the molar A-I/AII ratio of $\mathrm{Lp}(\mathrm{AI} w \mathrm{AII})$ ranges between 1.3 and 1.9. These ratios are consistent with earlier chemical cross-linking studies showing that $\mathrm{Lp}$ (AI w AII) particles with Stokes diameter between 7.8 and $9.7 \mathrm{~nm}$ have an apparent protein molecular weight equivalent to two molecules of A-I and one or two molecules of A-II per particle (27). The molar A-I/A-II ratio of the patient's Lp(AI w AII) was only 1 . Along with the small particle size $(<8.2 \mathrm{~nm})$, this suggests that the average Lp(AI w AII) in IM contained only one molecule of A-I and A-II per particle. In addition to $\mathrm{Lp}(\mathrm{AI} w \mathrm{AII})$ and $\mathrm{Lp}(\mathrm{AI} w / o \mathrm{AII})$, a third population of lipoprotein particles that is not found in normal subjects was present in the patient's plasma: $L p(A I I)$. These lipoproteins contained A-II as the major protein component, had a lipid/protein ratio only slightly less than normal HDL, and carried $60 \%$ of all the lipids located in her HDL. Chemical cross-linking studies showed that most of the Lp(A-II) molecules had an apparent protein molecular weight equivalent to four molecules of apo A-II. It is of interest to note that in vitro complexing of apo A-II with HDL lipids also resulted in HDL containing four molecules of A-II per particle (44). Since $\mathrm{Lp}$ (AII) contained $\sim 50 \%$ lipid and $50 \%$ A-II, it can be calculated that the average molecular mass of $\mathrm{Lp}(\mathrm{AII})$ is $\sim 139 \mathrm{kD}$, the size of small HDL.

All of the HDL particles in IM were rich in TG and poor in CE. Nonetheless, the FC/CE ratio and the proportion of core to surface lipid were normal, suggesting that most of the HDL particles were probably spherical and that lipid transfer protein activity was normal. The lipid/protein ratio $(25: 75 \%)$ of the patient's $\mathrm{Lp}$ (AI w AII) and Lp(AI w/o AII) was considerably lower than that of her relatives $(\sim 50: 50 \%)$ and other normolipidemic subjects we have studied $(7,9,30)$. Their size profiles showed substantial amounts of particles with Stokes diameters smaller than albumin. Thus, the A-I-containing HDL particles of IM were very dense and small lipoproteins, most of which would probably not be within the classical density 1.063-1.21 $\mathrm{g} / \mathrm{ml}$ HDL region. Similar small, spherical, and dense $\mathrm{Lp}$ (AI w AII ) and Lp (AI w/o AII) enriched with TG have been isolated from Hep G2 conditioned medium (45). Unlike the patient's A-I-containing lipoproteins, the lipid/protein ratio and particle size of $\mathrm{Lp}$ (AII) were nearly comparable to normal HDL. These lipoproteins would be predicted to float at density 1.21 $\mathrm{g} / \mathrm{ml}$ in the ultracentrifuge. Thus, the nature of the A-I- and/ or A-II-containing particles in IM appeared to be comparable to those in a subject with apo A-I deficiency heterozygous for a 45-bp deletion in exon 4 of the apo A-I gene (38) and in Tangier disease where most of the A-II was reported to be located in the plasma lipoproteins with alpha electrophoretic mobility whereas most of the A-I was found in the lipoprotein-free plasma fraction and migrated with prebeta mobility $(46,47)$.

HDL has been postulated to be involved in reverse cholesterol transport by stimulating intracellular cholesterol efflux from peripheral cells as well as in its subsequent extracellular transport. Recent studies from our laboratory (48) and others 
(49) have demonstrated that $\mathrm{Lp}$ (AI w/o AII) and $\mathrm{Lp}$ (AI w AII) are both equally effective at promoting efflux of intracellular and plasma membrane cholesterol from human skin fibroblasts, rabbit aortic smooth muscle cells, and FU5AH rat hepatoma cells. However, in a mouse adipocyte cell line, only $\mathrm{Lp}$ (AI w/o AII) were active promoters of cholesterol efflux whereas $\mathrm{Lp}$ (AI w AII ) inhibited the ability of $\mathrm{Lp}$ (AI w/o AII) in promoting cholesterol efflux (50). In the present study, intracellular cholesterol efflux was measured indirectly by the ability of $\mathrm{Lp}(\mathrm{AI})$ and $\mathrm{Lp}(\mathrm{AII})$ to decrease the pool of free cholesterol available for esterification in cholesterol-loaded human fibroblasts. Despite their abnormal lipid composition, both $\mathrm{Lp}(\mathrm{AI})$ and $\mathrm{Lp}$ (AII) were equally effective in decreasing cell cholesterol esterification in a dose-dependent manner. The patient's particles were as effective as the $\mathrm{Lp}(\mathrm{AI})$ fraction isolated from a control plasma when normalized for total protein and were apparently more effective when normalized for apo A-II concentration. These studies demonstrated for the first time that abnormal HDL particles of HDL-deficient subjects were effective in promoting intracellular cholesterol efflux. Furthermore, $L p(A)$ and $L p$ (AII) were equally effective in reducing intracellular cholesterol. Since this patient did not have elevated cholesterol and the HDL particles were effective promoters of cholesterol efflux, it is tempting to speculate that premature CAD may have resulted from a reduced total capacity of HDL to remove cholesterol from peripheral tissues.

The distribution of all four usually "HDL-associated" proteins was somewhat abnormal in the patient. First, most of her A-I and A-II were found in separate particles whereas, in normal individuals, approximately two thirds of A-I and essentially all A-II were associated with each other $(7,9,32)$. Second, in normal individuals and in the patient's relatives, $<1 \%$ of plasma A-I and A-II were found in the DS fraction; but in IM, 7\% of A-I and $12 \%$ A-II were located there. Third, in normal plasmas, $>85 \%$ of plasma apo D and LCAT are HDL associated $(7,51)$. However, in IM, $41 \%$ of apo D and $31 \%$ of LCAT were not associated with her HDL particles. The significance of the abnormal distribution of A-I, A-II, D, and LCAT in the patient's plasma is not clear. Increased association of A-I and/or A-II with the lower density lipoproteins has also been observed in Tangier plasma $(47,52)$. It has been suggested that in Tangier disease the increased presence of A-II in VLDL and LDL rendered those lipoproteins poor substrates for lipoprotein lipase (LPL) and may explain the hypertriglyceridemia in that disease (52). Hypertriglyceridemia, however, was not present in this patient, despite a low postheparin LPL activity. (Her postheparin plasma LPL activity, performed by Dr. J. D. Brunzell, University of Washington, was 54 compared with $220 \pm 59$ $\mathrm{nmol} \cdot \mathrm{min}^{-1} \cdot \mathrm{ml}^{-1}$ for normal controls.) Decreased association of LCAT with A-I-containing lipoproteins has also been observed in Tangier plasma and in Hep G2 conditioned medium $(45,53,54)$. Whether this decrease was the cause or the result of the abnormal HDL in the patient is not known. It is intriguing that despite the predominance of $\mathrm{Lp}(\mathrm{A}-\mathrm{II})$ in the patient and their apparently normal lipid composition minimal LCAT was found associated with these particles. Thus, the association of LCAT with HDL particles appears to be HDL subspecies specific.

In conclusion, an HDL-deficient patient with many clinical and biochemical manifestations of Tangier disease has been studied along with first-degree relatives. The patient's HDL was characterized by small, protein-rich $\mathrm{Lp}$ (AI w AII) and
$\mathrm{Lp}(\mathrm{AI} w / \mathrm{o} A \mathrm{AI})$ and the presence of substantial quantities of $\mathrm{Lp}$ (AII) with normal lipid/protein ratio. These particles were effective promoters of cholesterol efflux from cholesterolloaded fibroblasts. Although the genetic defect of the patient remains to be determined, the present findings are consistent with enhanced catabolism as the metabolic cause of HDL deficiency. Finally, because of the small family size, a causal relationship between HDL deficiency and CHD in this patient cannot be made.

\section{Acknowledgments}

The authors thank Dr. J. J. Albers and the Northwest Lipid Research Laboratories for performing lipid, apo B, and LCAT determinations; Ms. Casey Pierce for expert clinical help; and the patient and her family for their cooperation in this study.

This work was supported by National Institutes of Health Program Project Grant HL-30086 and by DK 35816, the Clinical Nutrition Research Unit at the University of Washington. A. J. Mendez was supported by a Nutrition, Lipid Metabolism and Arteriosclerosis Training Grant HL-07028, and HL-311943.

\section{References}

1. Heiss, G., and T. Tyroler. 1982. Are apolipoproteins useful for evaluating ischemic heart disease? A brief overview of the literature. Proc. Workshop Apolipoprotein Quantification. K. Lippel, editor. National Institutes of Health, Bethesda, MD. Publication No. 83-1266:7-22.

2. Gordon, D. J., J. L. Probstfield, R. J. Garrison, J. D. Neaton, W. P. Castelli, J. D. Knoke, D. R. Jacobs, S. Bangdiwala, and H. F. Tyroler. 1989. High density lipoprotein cholesterol and cardiovascular disease, four prospective American studies. Circulation 79:5-15.

3. Glomset, J. A. 1968. The plasma lecithin:cholesterol acyltransferase reaction. J. Lipid Res. 9:155-167.

4. Schaefer, E. J. 1984. Clinical, biochemical, and genetic features in familial disorders of high density lipoprotein deficiency. Arteriosclerosis. 4:303-322.

5. Stein, Y., M. C. Glangeaud, M. Fainaru, and O. Stein. 1975. The removal of cholesterol from aortic smooth muscle cells in culture and Landschutz ascites cells by fractions of human high-density lipoproteins. Biochim. Biophys. Acta. 380:106-118.

6. Oram, J. F., J. J. Albers, M. C. Cheung, and E. L. Bierman. 1981. The effects of subfractions of high density lipoprotein on cholesterol efflux from cultured fibroblasts. Regulation of low density lipoprotein receptor activity. J. Biol. Chem. 256:8348-8356.

7. Cheung, M. C., and J. J. Albers. 1984. Characterization of lipoprotein particles isolated by immunoaffinity chromatography: particles containing A-I and A-II and particles containing A-I but no A-II. J. Biol. Chem. 259:1220112209.

8. Cheung, M. C. 1986. Characterization of apolipoprotein A-containing lipoproteins. In Methods in Enzymology, Vol. 129 Albers, J. J. and J. Segrest, editors. Academic Press, Orlando, FL. 130-145.

9. Cheung, M. C., J. P. Segrest, J. J. Albers, J. T. Cone, C. G. Brouillette, B. H. Chung, M. Kashyap, M. A. Glasscock, and G. M. Anantharamaiah (1987). Characterization of high density lipoprotein subspecies: structural studies by single vertical spin ultracentrifugation and immunoaffinity chromatography. J. Lipid Res. 28:913-929.

10. Lipid and lipoprotein analysis. In Manual of Laboratory Operations, Lipid Research Clinics Program. 2nd ed. A. Hainline, J. Karon, and K. Lippel, editors. Public Health Service, National Institutes of Health, Bethesda, MD.

11. Warnick, G. R., J. Benderson, and J. J. Albers. 1982. Dextran sulfate$\mathrm{Mg}^{2+}$ precipitation procedure for quantitation of high-density-lipoprotein cholesterol. Clin. Chem. 28:1379-1388.

12. Friedewald, W. T., R. I. Levy, and D. S. Frederickson. 1972. Estimation of the concentration of low-density lipoprotein cholesterol in plasma, without use of the preparative ultracentrifugation. Clin. Chem. 18:499-502.

13. Yokoyama, S., R. Hayashi, M. Satani, and A. Yamamoto. 1985. Selective removal of low density lipoprotein by plasmapheresis in familial hypercholesterolemia. Arteriosclerosis. 5:613-622.

14. Weisgraber, K. H., and R. W. Mahley. 1980. Subfraction of human high density lipoproteins by heparin sepharose affinity chromatography. J. Lipid Res. 21:316-325.

15. Brinton, E. A., J. F. Oram, C. H. Chen, J. J. Albers, and E. L. Bierman. 1986. Binding of high density lipoprotein to cultured fibroblasts after chemical alteration of apoprotein amino acid residue. J. Biol. Chem. 261:495-503. 
16. Oram, J. F. 1986. Receptor-mediated transport of cholesterol between cultured cells and high density lipoproteins. Methods Enzymol. 129:645-659.

17. Warnick, G. R. 1986. Enzymatic methods for the quantification of lipoprotein lipids. Methods Enzymol. 129:101-123.

18. Albers, J. J., P. W. Wahl, V. G. Cabana, W. R. Hazzard, and J. J. Hoover. 1976. Quantitation of apolipoprotein A-I of human plasma high density lipoprotein. Metabolism. 25:633-644.

19. Cheung, M. C., and J. J. Albers. 1977. The measurement of apolipoprotein A-I and A-II levels in men and women by immunoassay. J. Clin. Invest. 60:43-50.

20. Albers, J. J., V. G. Cabana, and W. R. Hazzard. 1975. Immunoassay of human plasma apolipoprotein B. Metabolism. 24:1339-1351.

21. Albers, J. J., M. C. Cheung, S. L. Ewens, and J. H. Tollefson. 1981. Characterization and immunoassay of apolipoprotein D. Atherosclerosis. 39:395-409.

22. Albers, J. J., J. L. Adolphson, and C.-H. Chen. 1981. Radioimmunoassay of human plasma lecithin:cholesterol acyltransferase. J. Clin. Invest. 67:141-148.

23. Chen, C.-H., and J. J. Albers. 1982. Characterization of proteoliposomes containing apoprotein A-I: a new substrate for the measurement of lecithin:cholesterol acyltransferase activity. J. Lipid Res. 23:680-691.

24. Laemmli, U. K. 1970. Cleavage of structural proteins during the assembly of the head of the bacteriophage. Nature (Lond.). 227:680-685.

25. Nichols, A. V., P. J. Blanche, and E. L. Gong. 1983. Gradient gel electrophoresis of human plasma high density lipoproteins. In Handbook on Electrophoresis. Lewis, L. A., editor. Vol. III. CRC Press, Boca Raton, FL. 29-47.

26. Swaney, J. B., and K. O'Brien. 1978. Cross-linking studies of the self-association properties of apo-A-I and apo-A-II from human high density lipoprotein. J. Biol. Chem. 253:7069-7077.

27. Cheung, M. C., A. V. Nichols, P. J. Blanche, E. L. Gong, G. Francheschini, and C. R. Sirtori. 1988. Characterization of A-I-containing lipoproteins in subjects with A-I $\mathrm{I}_{\text {Milano }}$ variant. Biochim. Biophys. Acta. 960:73-82.

28. Lipid Research Clinics Program Epidemiology Committee. 1979. Plasma lipid distributions in selected North American populations: the Lipid Research Clinic Program prevalence study. Circulation. 60:427-439.

29. Towbin, H., T. Stachelin, and J. Gordon. 1979. Electrophoretic transfer of proteins from polyacrylamide gels to nitrocellulose sheets: procedure and some applications. Proc. Natl. Acad. Sci. USA. 76:4350-4354.

30. Cheung, M. C., and A. C. Wolf. 1988. Differential effect of ultracentrifugation on apolipoprotein A-I-containing lipoprotein subpopulations. J. Lipid Res. 29:15-25.

31. James, R. W., D. Hochstrasser, J.-D. Tissot, M. Funk, R. Appel, F. Barja C. Pellegrini, A. F. Muller, and D. Pometta. 1988. Protein heterogeneity of lipoprotein particles containing apolipoprotein A-I without apolipoprotein A-II and apolipoprotein A-I with apolipoprotein A-II isolated from human plasma. $J$. Lipid Res. 29:1557-1571.

32. Cheung, M. C. 1987. Characterization and distribution of HDL subpopulations. In Proc. Workshop Lipoprotein Heterogeneity. K. Lippel, editor. NIH Publication No. 87-2646. 341-349.

33. Heinen, R. J., P. N. Herbert, D. S. Frederickson, T. Forte, and F. T. Lindgren. 1978. Properties of the plasma very low and low density lipoproteins in Tangier disease. J. Clin. Invest. 61:120-132.

34. Rall, S. C., K. H. Weisgraber, R. W. Mahley, C. Ehnholm, O. Schamaun, B. Olaisen, J. P. Blomhoff, and P. Teisberg. 1986. Identification of homozygosity for human apolipoprotein A-I variant. J. Lipid Res. 27:436-441.

35. Forte, T. M., A. V. Nichols, and R. M. Krauss. 1984. Familial apolipoprotein AI and apolipoprotein CIII deficiency. J. Clin. Invest. 74:1601-1613.

36. Schaefer, E. J., J. M. Ordovas, S. W. Law, et al. 1985. Familial apolipoprotein A-I and C-III deficiency variant II. J. Lipid Res. 26:1089-1101.

37. Funke, H., A. von Eckardstein, P. H. Hayden, M. Karas, J. Albers, and G. Assman. 1991. A frameshift mutation in the human apolipoprotein A-I gene causes high density lipoprotein deficiency, partial lecithin:cholesterol-acyltransferase deficiency, and corneal opacities. J. Clin. Invest. 87:371-376.

38. Deeb, S. S., M. C. Cheung, R. Peng, A. Wolf, R. Stern, J. J. Albers, and R. H. Knopp. 1991. A mutation in the human apolipoprotein A-I gene: dominant effect on the level and characteristics of plasma HDL. J. Biol. Chem. 266:13654-13660.

39. Funke, H., A. von Eckardstein, P. H. Pritchard, J. J. Albers, J. J. P. Kastelein, C. Droste, and G. Assmann. 1991. A molecular defect causing fish eye disease: an amino acid exchange in lecithin-cholesterol acyltransferase (LCAT) leads to the selective loss of $\alpha$-LCAT activity. Proc. Natl. Acad. Sci. USA. 88:4855-4859.

40. Weisgraber, K. H., T. P. Bersot, R. W. Mahley, G. Francheschini, and

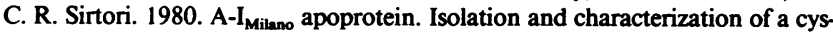
teine-containing variant of the A-I apoprotein from human high density lipoproteins. J. Clin. Invest. 66:901-907.

41. Utermann, G., J. Haas, A. Steinmetz, R. Paetzold, S. C. Rall, Jr., K. H. Weisgraber, and R. W. Mahley. 1984. Apolipoprotein A-I Giessen (Pro ${ }^{143}-$ Arg), a mutant that is defective in activating lecithin:cholesterol acyltransferase. Eur. J. Biochem. 144:325-331.

42. Schamaun, O., B. Olaisen, T. Gedde-Dahl, Jr., and P. Teisberg. 1983. Genetic studies of an apo A-I lipoprotein variant. Hum. Genet. 64:380-383.

43. Utermann, G., A. Steinmetz, R. Paetzold, J. Wilk, G. Feussner, H. Kaffarnik, C. Mueller-Eckhardt, D. Seidel, K.-H. Vogelberg, and F. Zimmer. 1982. Apolipoprotein A-I $\mathrm{I}_{\text {Mrturg }}$. Studies on two kindreds with a mutant of human apolipoprotein A-I. Hum. Genet. 62:329-337.

44. Ritter, M. C., and A. M. Scanu. 1979. Apolipoprotein A-II and structure of human high density lipoproteins, an approach by reassembly techniques. $J$. Biol. Chem. 254:2517-2525.

45. Cheung, M. C., K. D. Lum, C. G. Brouillette, and C. L. Bisgaier. 1989. Characterization of apo A-I-containing lipoprotein subpopulations secreted by HepG2 cells. J. Lipid Res. 30:1429-1436.

46. Assmann, G., P. N. Herbert, D. S. Fredrickson, and T. Forte. 1977. Isolation and characterization of an abnormal high density lipoprotein in Tangier disease. J. Clin. Invest. 60:242-252.

47. Assmann, G., E. Smootz, K. Adler, A. Capurso, and K. Oette. 1977. The lipoprotein abnormality in Tangier disease. Quantitation of A apoproteins. $J$. Clin. Invest. 59:565-575.

48. Oikawa, S., A. J. Mendez, M. C. Cheung, J. F. Oram, and E. L. Bierman. 1991. Effect of apo A-I and A-I/A-II HDL particles on intracellular cholesterol efflux. Arteriosclerosis. 11:1605a.

49. Johnson, W. J., E. P. C. Kilsdonk, A. van Tol, M. C. Phillips, and G. H. Rothblat. 1991. Cholesterol efflux from cells to immunopurified subfractions of human high density lipoprotein: Lp-AI and Lp-AI/AII. J. Lipid Res. 32:19932000.

50. Barbaras, R., P. Puchois, J. C. Fruchart, and G. Ailhaud. 1987. Cholesterol efflux from cultured adipose cells is mediated by $\mathrm{Lp}(\mathrm{AI})$ particles but not by Lp(AI:AII) particles. Biochem. Biophys. Res. Commun. 142:63-69.

51. Cheung, M. C., A. C. Wolf, K. D. Lum, J. H. Tollefson, and J. J. Albers. 1986. Distribution and localization of lecithin:cholesterol acyltransferase and cholesteryl ester transfer activity in A-I-containing lipoproteins. J. Lipid Res. 27:1135-1144.

52. Wang, C.-S., P. Alaupovic, R. E. Gregg, and H. B. Brewer, Jr. 1987. Studies on the mechanism of hypertriglyceridemia in Tangier disease. Determination of plasma lipolytic activities, $\mathbf{k}_{1}$ values and apolipoprotein composition of the major lipoprotein density classes. Biochim. Biophys. Acta. 920:9-19.

53. Cheung, M. C., D. Wang, K. D. Lum, and J. A. Albers. 1988. Cholesterol esterification by lecithin-cholesterol acyltransferase in A-I-free plasma. Biochim. Biophys. Acta. 962:258-264.

54. Pritchard, P. H., R. McLeod, J. Frohlich, M. C. Park, B. J. Kudchodkar, and A. G. Lacko. 1988. Lecithin:cholesterol acyltransferase in familial HDL deficiency (Tangier disease). Biochim. Biophys. Acta. 958:227-234. 